\title{
Impaired global and segmental myocardial deformation assessed by two-dimensional speckle tracking echocardiography in patients with vitamin B12 deficiency
}

\author{
Zekeriya Kaya ${ }^{1}$, Asuman Bicer ${ }^{1}$, Funda Yalcin ${ }^{2}$, Arzu Er ${ }^{3}$, \\ Aysun Camuzcuoglu ${ }^{4}$, Nurhan Korkmaz ${ }^{2}$, Yusuf Sezen ${ }^{1}$ \\ ${ }^{1}$ Department of Cardiology, Harran University School of Medicine, Turkey \\ ${ }^{2}$ Department of Chest Diseases, Harran University School of Medicine, Turkey \\ ${ }^{3}$ Department of Cardiology, Ministry of Health Tokat Government Hospital, Turkey \\ ${ }^{4}$ Department of Obstetrics and Gynecology, Harran University School of Medicine, Turkey
}

\begin{abstract}
Background: Contrary effects of vitamin B12 deficiency have been shown on the cardiovascular system. Aim of our study was to analyze left ventricular (LV) myocardial deformation, by using the two dimensional (2D) speckle tracking echocardiography (STE) in patients with vitamin B12 deficiency and normal LV ejection fraction.

Methods: Twenty-five patients with vitamin B12 deficiency (B12 levels $<200 \mathrm{pg} / \mathrm{mL}$; mean age: $29.6 \pm 8.2$ years, 15 female), and 27 healthy controls (B12 levels $>200 \mathrm{pg} / \mathrm{mL}$; mean age: $30.1 \pm 6.9$ years, 13 female) were included in the study. $2 D$ echocardiography images were transferred to a workstation for further offline analysis. Longitudinal peak systolic (LPSS) and global strain (LGS) was obtained from 4 chamber and apical long axis (APLAX) views.

Results: Standard echocardiographic parameters and tissue Doppler imaging (TDI) velocities were compared between the groups. All LPSS values in the patient group except for apical $4 C$ septal wall longitudinal strain were significantly decreased than those in the control group. There was a positive correlation between B12 levels and strain values except apical $4 C$ septal wall strain values.

Conclusions: We found that in patients with vitamin B12 deficiency, global and segmental myocardial deformation was impaired and this impairment was correlated with the levels of vitamin B12. (Cardiol J 2014; 21, 1: 60-66)
\end{abstract}

Key words: left ventricular myocardial deformation, speckle tracking echocardiography, strain, vitamin B12

\section{Introduction}

Vitamin B12 (cobalamin) which functions as a coenzyme in two metabolic processes - the conversion of methylmalonyl-CoA to succinyl-CoA and the remethylation of homocysteine to methionine
— is an essential nutrient for proliferation and metabolism of normal cell activity [1-4]. Vitamin B12 deficiency is common and is one of the major public health problems. The diagnosis of vitamin B12 deficiency has traditionally been based on low serum vitamin B12 levels, usually less than $200 \mathrm{pg} / \mathrm{mL}$

Address for correspondence: Dr Asuman Bicker, Associate Professor, Department of Cardiology, Harran University School of Medicine, Turkey, tel: +90 414318 3159, fax: 414318 3192, e-mail: asubicer@yahoo.com 
(150 pmol/L), along with clinical evidence of disease. In the case of deficiency, conversion of methylmalonyl-CoA is blocked (inside the mitochondria), which in turn can result in increased lipogenesis [2]. Vitamin B12 deficiency impairs fatty acid oxidation and energy metabolism in heart through epigenomic mechanisms related to imbalanced acetylation/methylation. In addition, decreased vitamin B12 is associated with increased plasma homocysteine levels. High homocysteine level in blood (hyperhomocysteinemia), which is an independent risk factor for atherosclerotic disease, disrupts endothelial functions and causes activation of procoagulant factors, malfunction of anticoagulant mechanisms [5]. Hyperhomocysteinemia has been suggested as a risk factor for cardiovascular disease, blood clotting abnormalities, atherosclerosis, myocardial infarction and ischemic stroke [5].

Further, anemia due to vitamin B12 deficiency may result in heart failure (HF). Low hemoglobin levels in anemia are associated with an elevated cardiac output at rest, cardiomegaly, and, frequently, heart murmurs, due to an increase in stroke volume [6]. It has been postulated that excessive work load chronically imposed on heart by the cardiac output may result in left ventricle (LV) dysfunction. LV diastolic dysfunction occurs earlier in the development of overall cardiac dysfunction, and its presence is an independent risk factor for mortality among adults with anemia [7]. Therefore, it is very likely that B12 deficiency affects cardiovascular system partly through hyperhomocysteinemia and partly through its direct effects.

Echocardiography is an important diagnostic tool for the detection of HF. With the echocardiographic methods developed in recent years decreased heart function can be determined before the occurrence of clinical HF and with the heart's structure remaining yet intact. Two dimensional (2D) speckle tracking echocardiography (STE) has recently emerged as a novel echocardiographic technique for rapid, bedside the analysis of regional LV strains in the longitudinal, radial, and circumferential directions [8]. Subclinical changes in LV function in anemia can be identified by quantifying myocardial strain, a dimensionless measurement of deformation, expressed as a fractional or percentile change from an object's original dimension [9]. 2D STE has been shown to provide complementary information about the clinical assessment of cardiac function $[10,11]$. It may assess myocardial function and detect early subclinical myocardial involvement in many heart diseases, as well as quantify regional myocardial function in ischemic heart disease.
Assessment of regional LV function with 2D STE in patients with vitamin B12 deficiency has not yet been studied. The aim of our study was to assess the ability of subtle differences in the LV strain patterns in the longitudinal directions to characterize features of subclinical LV dysfunction in patients presenting with B12 deficiency.

\section{Methods}

\section{Study population}

Evaluated at different clinics of our hospital, 27 control subjects (group I, 13 female, mean age $30.1 \pm 6.9$ years; B12 levels $>200 \mathrm{pg} / \mathrm{mL}$ ) with normal vitamin B12 levels and 25 patients (group II, 15 female, mean age $29.6 \pm 8.2$ years) with similar characteristics but with vitamin B12 deficiency $(<200 \mathrm{pg} / \mathrm{mL})$ were included in the study. Patients with established coronary artery disease, echocardiography evidence of regional or global wall motion abnormalities, atrial fibrillation, valvular heart disease, diabetes mellitus, hypertension (systolic blood pressure $\geq 140 \mathrm{~mm} \mathrm{Hg}$, diastolic blood pressure $\geq 90 \mathrm{~mm} \mathrm{Hg}$ or drug use for the disease), chronic obstructive airway disease, renal failure, hypertrophic cardiomyopathy, patients receiving replacement therapy with vitamin B12 previously, and anemia for different reasons like folate or iron deficiency were excluded from the study.

Study protocol was approved by Institutional Ethics Review Board and conforms to the principles stated in the Declaration of Helsinki. All the patients were informed about the procedure to be performed, and their written consents were obtained.

\section{Echocardiographic measurements}

Echocardiographic examinations were performed in all cases using Vivid E9 Dimension echocardiography device (Vingmed Ultrasound, GE, Horten, Norway) with $3.5 \mathrm{MHz}$ single matrix transducer while the patient was lying in the left lateral decubitus position. All the images were stored in digital media (Echopac Workstation; Vingmed Ultrasound GE) for later measurements. Echocardiographic recordings were obtained from standard parasternal long and short axis, apical long axis (APLAX) and apical 4 chamber (4C) views. Echocardiographic evaluation and measurements were performed according to the guidelines of American Society of Echocardiography [12]. Left atrium, LV end-diastolic and end-systolic, and aortic root dimensions were measured from parasternal long-axis view. The LV 
volumes and ejection fraction were obtained by the modified biplane Simpson method [12]. Mitral and tricuspid inflow recordings were taken by pulsed wave Doppler ultrasound. From these recordings diastolic $\mathrm{E}$, and $\mathrm{A}$ wave velocities were measured and $\mathrm{E} / \mathrm{A}$ was calculated.

\section{Tissue Doppler echocardiography}

During imaging in the apical $4 \mathrm{C}$ view, tissue velocity mode of the device was selected, and pulsed wave Doppler sample volume was placed at the level of lateral mitral rings and TDE images were obtained. Myocardial peak systolic (S), early diastolic (E'), late diastolic (A') velocities were measured [13] and E/E' was calculated.

\section{D speckle tracking echocardiography}

2D echocardiography images were obtained from APLAX and apical $4 \mathrm{C}$ views. All images were obtained during breath hold, and stored in cine-loop format from three consecutive beats. The frame rate for images was between 40 and $80 \mathrm{frames} / \mathrm{s}$. All data were transferred to a workstation for further offline analysis. After defining the endocardial border manually, an epicardial tracing was automatically developed by the software system for each view. If the automatically obtained tracking segments were adequate for analysis, the software system was allowed to read the data, whereas analytically inadequate tracking segments were either corrected manually or excluded from the analysis. Strain measurements were reported as the longitudinal peak systolic (LPSS) and global strain (LGS) automatically developed by the software system for APLAX and $4 \mathrm{C}$ views.

For the assessment of intra-observer variability, the analyses were repeated twice by the same observer within 1 week. For the inter-observer variability assessment, a second independent observer repeated the analyses.

\section{Statistical evaluation}

For statistical evaluation SPSS 11.5 (SPSS Inc. Chicago USA) statistical software program was used. Continuous variables were expressed as means $\pm \mathrm{SD}$, and categorical variables as percentages. One sample Kolmogorov-Smirnov test was used for the normality test of all variables. For the comparison of parametric variables independent sample $t$ test, and for categorical variables $\chi^{2}$ test were used. Binary logistic regression analysis was performed to identify the independent predictors of strain values in the groups. Standardized $\beta$-regression coefficients and their significance from logistic regression analysis were reported. Correlations between variables were tested by Pearson correlation tests. Linear regression analysis was performed to identify the independent predictors of global strain by including the parameters which were correlated with the global strain in bivariate correlation analysis. Standardized $\beta$-regression coefficients and their significance from linear regression analysis were reported. In all assessments 2 -sided $\mathrm{p}<0.05$ was accepted as statistically significant.

\section{Results}

A total of 50 patients with B12 deficiency were evaluated. Seven patients who did not have a good echocardiographic window or analytically inadequate tracking segments, 4 patients with cardiac involvement, 3 patients with arrhythmia and 11 patients with additional iron or folate deficiency were excluded from the study. A total of 25 patients with B12 deficiency and 27 sex- and age-matched controls were included in the study. Baseline clinical and demographic characteristics of both groups and their comparisons are summarized in Table 1 . There were no statistically significant differences between the two groups with regard to age, gender, smoking, systolic/diastolic blood pressure and hyperlipidemia. There was statistically significant difference between the groups regarding B12 levels $(264.85 \pm 50.24 \mathrm{pg} / \mathrm{mL}$ in group I and $136.44 \pm 31.95 \mathrm{pg} / \mathrm{mL}$ in group II, $\mathrm{p}<0.001$ ) (Table 1). All the laboratory characteristics between groups except for B12 levels were also similar. Standard echocardiographic parameters and tissue Doppler imaging (TDI) velocities were comparable between the groups (Table 2). All LPSS values in the patient group except for apical $4 \mathrm{C}$ septal wall longitudinal strain were significantly decreased co pared to those in the control group (Table 3 ). There was a positive correlation between B12 levels and strain values except for septal wall strain values (Table 4). There was no correlation of LPSS and LGS values with other demographic, clinical and laboratory variables ( $p>0.05$ for all) (data not shown). Independent predictors of plasma B12 levels were determined with linear regression analysis by including the parameters that were correlated with the strain values in correlation analysis and neither of the strain values was detected as an independent predictor of plasma B12 levels (Table 4). 
Table 1. General characteristics in controls and patients.

\begin{tabular}{|c|c|c|c|}
\hline & Controls $(n=27)$ & Patients $(n=25$ ) & $\mathbf{P}$ \\
\hline Age [years] & $30.1 \pm 6.9$ & $29.0 \pm 8.0$ & 0.808 \\
\hline Female sex & $13(48 \%)$ & $15(60 \%)$ & 0.419 \\
\hline BMI $\left[\mathrm{kg} / \mathrm{m}^{2}\right]$ & $25.7 \pm 4.4$ & $27.8 \pm 6.1$ & 0.165 \\
\hline $\mathrm{SBP}[\mathrm{mm} \mathrm{Hg}]$ & $115.0 \pm 16.5$ & $113.8 \pm 18.5$ & 0.854 \\
\hline $\mathrm{DBP}[\mathrm{mm} \mathrm{Hg}]$ & $71.7 \pm 11.1$ & $71.7 \pm 13.0$ & 1.000 \\
\hline Dyslipidemia & $6(22 \%)$ & $3(12 \%)$ & 0.330 \\
\hline Smoking & $11(40 \%)$ & $6(24 \%)$ & 0.238 \\
\hline Fasting blood glucose [mg/dL] & $94.6 \pm 20.5$ & $98.3 \pm 20.5$ & 0.516 \\
\hline Blood urea nitrogen [mg/dL] & $26.1 \pm 8.7$ & $22.0 \pm 6.6$ & 0.064 \\
\hline Serum creatinine $[\mathrm{mg} / \mathrm{dL}]$ & $0.74 \pm 0.19$ & $0.65 \pm 0.17$ & 0.093 \\
\hline Hemoglobin [g/dL] & $14.3 \pm 1.7$ & $13.3 \pm 2.2$ & 0.059 \\
\hline Mean corpuscular volume [fL] & $84.9 \pm 4.7$ & $83.1 \pm 3.7$ & 0.141 \\
\hline Platelet count $\left[10^{3} / \mu \mathrm{L}\right]$ & $244.3 \pm 53.4$ & $242.1 \pm 65.3$ & 0.895 \\
\hline White blood cells count $\left[10^{3} / \mu \mathrm{L}\right]$ & $9.63 \pm 2.85$ & $9.49 \pm 3.65$ & 0.884 \\
\hline Serum levels of vitamin B12 [pg/mL] & $264.9 \pm 50.2$ & $136.4 \pm 32.0$ & $<0.001$ \\
\hline
\end{tabular}

Statistically significant results are given in bold; BMI — body mass index; SBP — systolic blood pressure; DBP — diastolic blood pressure

Table 2. Standard echocardiographic parameters in controls and patients.

\begin{tabular}{lccc}
\hline & Controls $(\mathbf{n}=\mathbf{2 7})$ & Patients $(\mathbf{n}=\mathbf{2 5})$ & P \\
\hline LVEDV [mL] & $100.4 \pm 18.4$ & $102.9 \pm 26.6$ & 0.699 \\
LVESV [mL] & $31.0 \pm 8.4$ & $32.6 \pm 11.9$ & 0.582 \\
Stroke volume [mL] & $69.4 \pm 13.4$ & $70.2 \pm 17.6$ & 0.862 \\
LVEF [\%] & $69.2 \pm 5.4$ & $68.4 \pm 7.0$ & 0.652 \\
Fractional shortening [\%] & $39.0 \pm 4.4$ & $38.4 \pm 5.8$ & 0.655 \\
Mitral E peak velocity [cm/s] & $88.5 \pm 15.4$ & $85.8 \pm 14.7$ & 0.522 \\
Mitral A peak velocity [cm/s] & $64.2 \pm 15.9$ & $65.8 \pm 11.0$ & 0.688 \\
Mitral E/A ratio & $1.44 \pm 0.34$ & $1.33 \pm 0.27$ & 0.204 \\
Tissue Doppler, lateral S [cm/s] & $11.5 \pm 2.3$ & $12.2 \pm 2.6$ & 0.294 \\
Tissue Doppler, lateral E' [cm/s] & $17.4 \pm 2.4$ & $16.3 \pm 3.3$ & 0.184 \\
Tissue Doppler, lateral A' [cm/s] & $9.63 \pm 2.45$ & $9.29 \pm 2.56$ & 0.633 \\
E/E' ratio & $5.15 \pm 0.95$ & $5.40 \pm 0.95$ & 0.385 \\
\hline
\end{tabular}

LVEDV — left ventricular end-diastolic volume; LVESV — left ventricular end-systolic volume; LVEF — left ventricular ejection fraction

\section{Discussion}

For the first time, our study evaluated LV myocardial functions in patients with B12 deficiency by $2 \mathrm{D}$ STE in addition to conventional echocardiographic examination. We found that global and segmental longitudinal myocardial deformation was impaired and this impairment was correlated with the levels of vitamin B12 in patients with B12 deficiency, though we could not reveal independent association of vitamin B12 levels and strain values in the linear regression analysis (Table 4).

Inadequate dietary intake, inability to absorb from the intestinal tract, and metabolic and trans- port disorders which result from being unable to use the vitamin may cause the lack of B12. The incidence of B12 deficiency appears to increase with age. HIV-infected and vegetarians who are not taking in proper amounts of B12 are also prone to deficiency [3]. We do not know the reason for vitamin deficiency in this younger age group in our study, though absorption disorders associated with genetic factors, bacterial infections and parasitic infestations would be plausible mechanisms.

In our study we found that all LPSS values in the patient group except for apical $4 \mathrm{C}$ septal wall longitudinal strain were significantly decreased compared to those in the control group, and there 
Table 3. Comparison of left ventricle longitudinal peak systolic and global strain values in controls and patients and regression analysis between the groups.

\begin{tabular}{|c|c|c|c|c|c|}
\hline & $\begin{array}{l}\text { Controls } \\
(n=27)\end{array}$ & $\begin{array}{l}\text { Patients } \\
(\mathrm{n}=25)\end{array}$ & $\mathbf{P}$ & $\begin{array}{c}\text { Beta regression } \\
\text { coefficient }\end{array}$ & $\mathbf{P}$ \\
\hline $\begin{array}{l}\text { LPSS, apical } \\
4 C \text {, lateral wall }\end{array}$ & $-19.90 \pm 4.53$ & $-13.86 \pm 4.58$ & $<0.001$ & -0.217 & 0.187 \\
\hline $\begin{array}{l}\text { LPSS, apical } \\
4 C \text {, septal wall }\end{array}$ & $-20.19 \pm 3.75$ & $-19.88 \pm 3.35$ & 0.753 & & \\
\hline LGS, apical 4C & $-20.58 \pm 3.23$ & $-17.45 \pm 3.58$ & 0.002 & -193.984 & 0.999 \\
\hline $\begin{array}{l}\text { LPSS, APLAX, } \\
\text { posterior wall }\end{array}$ & $-20.09 \pm 3.77$ & $-16.92 \pm 3.85$ & 0.004 & -0.223 & 0.423 \\
\hline $\begin{array}{l}\text { LPSS, APLAX, } \\
\text { septal wall }\end{array}$ & $-20.41 \pm 3.02$ & $-17.02 \pm 5.16$ & 0.005 & -0.377 & 0.088 \\
\hline LGS, APLAX & $-20.80 \pm 2.94$ & $-17.62 \pm 3.56$ & 0.001 & -193.689 & 0.999 \\
\hline
\end{tabular}

Binary logistic regression analysis was performed to identify the independent predictors of strain values; standardized $\beta$-regression coefficients and their significance from logistic regression analysis were reported; statistically significant results are given in bold; $p<0.05$ was considered statistically significant; LPSS — longitudinal peak systolic strain; 4C — four chamber; LGS - longitudinal global strain;

APLAX - apical long axis

Table 4. Correlation and regression analysis between B12 levels and strain values.

\begin{tabular}{lcccc} 
& $\begin{array}{c}\text { Pearson correlation } \\
\text { coefficient }\end{array}$ & $\mathbf{P}$ & $\begin{array}{c}\text { Beta regression } \\
\text { coefficient }\end{array}$ & P \\
\hline LPSS, apical 4C, lateral wall & 0.457 & $\mathbf{0 . 0 0 1}$ & 5.235 & 0.077 \\
LPSS, apical 4C, septal wall & -0.008 & 0.953 & & 0.564 \\
LGS, apical 4C & 0.341 & $\mathbf{0 . 0 1 3}$ & 5.235 & 0.512 \\
LPSS, APLAX, posterior wall & 0.301 & $\mathbf{0 . 0 3 2}$ & $\mathbf{0 . 2 2 6}$ & 0.078 \\
LPSS, APLAX, septal wall & 0.356 & $\mathbf{0 . 0 1 0}$ & 114.36 & 0.086 \\
LGS, APLAX & 0.368 & $\mathbf{0 . 0 0 7}$ & & \\
\hline
\end{tabular}

Pearson correlation coefficients were used for comparing continuous variables and strain values; linear regression analysis was performed to identify the independent predictors of strain values; standardized $\beta$-regression coefficients and their significance from linear regression analysis were reported; statistically significant results are given in bold; $p<0.05$ was considered statistically significant; LPSS - longitudinal peak systolic strain; 4C — four chamber; LGS — longitudinal global strain; APLAX — apical long axis

was a positive correlation between B12 levels and strain values except apical $4 \mathrm{C}$ septal wall strain values. Further prospective studies with larger sample size are needed to elucidate the mechanism of non-uniform myocardial deformation of the absence of serum vitamin B12 levels and interventricular septum strain association.

LV myocardial dysfunction, even though subclinical, is associated with worse prognosis because of leading to increased risk of cardiovascular complications detected in various diseases $[14,15]$. Since standard echocardiography, with the assessment of LVEF and pulse wave Doppler mitral inflow parameters is often inadequate to identify early myocardial abnormalities, other diagnostic tools are needed in order to detect the onset of cardiac involvement [16-18]. With this respect, TDI and, more recently, 2D strain, have been shown to provide a more accurate evaluation of myocardial function. Tissue velocity measurements by TDI at the level of mitral and tricuspid annulus have been used for the diagnosis and monitoring of many cardiac diseases with higher sensitivity, and specificity $[13,19]$. However, Doppler-only-based techniques are limited due to angle dependence of the signal and TDI has a limited utility in the segmental and regional evaluation of myocardial functions because of technical drawbacks. 2D STE techniques overcome some of these limitations by allowing tracking in any direction within the 2D image. Computerized 2D STE is a new method that provides quantitative data during evaluation process of segmental, regional, and global LV functions with apparent advantages over other echocardiography-based methods used in daily practice for LV systolic or diastolic function analysis with regard to angle independency and temporal and spatial resolution, notwithstanding 2D STE is inferior to tissue velocity imaging derived strain [20-22]. Our findings support these 
data as we observed impaired longitudinal peak systolic strain in spite of preserved and comparable echocardiographic parameters including TDI velocities between the groups. However, the mechanism for spared diastolic function in case of affected systolic myocardial function in vitamin B12 deficiency remains to be established though we have not evaluated diastolic functions by $2 \mathrm{D}$ STE which was beyond our aim and it may be one of the limitations of our study.

Several studies have been reported on the impact of vitamin B12 on cardiovascular system. Taban-Shomal et al. [23] studied "the cardiac effects of prolonged vitamin B12 and folate deficiency in rats". They found that fractional shortening, LV dimension at end-diastole and end-systole, posterior wall thickness, perivascular collagen, mast cell number, and natriuretic peptide tissue levels were comparable in vitamin deficiency and control animals. Interstitial collagen, plasma natriuretic peptides, and tissue homocysteine levels were decreased in vitamin deficient animals. They concluded that vitamin B12 deficiency did not affect cardiac function and morphology. There is only very limited evidence that vitamin B12 deficiency predisposes to the risk of mortality and morbidity from cardiovascular diseases in adults. Conflicting effects of vitamin B12 deficiency on the cardiovascular system have been shown [24-29]. Looker et al. [25] and Zeitlins' et al. [26] studies showed an untoward effect of vitamin B12 that increased vitamin B12 levels were positively associated with increased total mortality and combined fatal and non-fatal cardiac disease. In another study there was no association between B12 concentrations and death from coronary heart disease or cardiovascular disease in a cohort without any cardiovascular disease at baseline [24]. Though our study is not a cardiovascular outcome study, with the findings of the present study we have revealed that the decreased serum vitamin B12 level is associated with subtle cardiac involvement evidenced with impaired strain values. Despite the link between the presence of vitamin B12 deficiency and strain values and significant association of serum vitamin B12 levels and strain values, we could not reveal independent asso ciation between the above-mentioned parameters. Several issues might have led to the absence of this independent association such as young study population $(30.1 \pm 6.9$ years in group I and $29.6 \pm 8.2$ years in group II) with decreased overall cardiovascular risk, duration of vitamin B12 deficiency (which might be fairly short in our young study population), and small sample size. Gueant Rodriguez et al. [30] showed that homocysteine predicts increased natriuretic peptides which are quantitative markers of cardiac dysfunction through a link with impaired mitochondrial fatty oxidation. Abnormally elevated plasma natriuretic peptides therefore provide independent evidence that a patient has cardiac dysfunction in spite of preserved LV ejection fraction.

\section{Limitations of the study}

One of the limitations of our study was that homocysteine or methylmalonic acid levels which would add to the value of our manuscript and associated with true low B12 levels were not investigated. Additionally, it should be noted that the present study had a relatively small sample size and larger sample size is needed to confirm/exclude the findings of the study. Furthermore, the apical 2 chamber views were inadequate to analyze in our imaging archive. Therefore, we could not analyze them and we could not calculate the global strain which was based on averaging three apical views traditionally. Since longitudinal strain values were more sensitive than other deformation values like radial or circumferential, we only studied the longitudinal strain. However, it would be better to show radial or circumferential deformations beside longitudinal fibers. Despite these limitations, this study brings attention to a novel, important hypothesis at the forefront of cardiology research by showing an association between LV myocardial deformation and B12 levels and also by determining subclinical $\mathrm{LV}$ dysfunction at an early stage using $2 \mathrm{D}$ strain as diagnostic tool.

\section{Conclusions}

Consequently, we detected that myocardial strain values were decreased in patients with vitamin B12 deficiency. Further prospective studies with longer follow-up periods and larger sample size are needed to confirm/exclude the findings of this study and to further elucidate its clinical importance.

\section{Acknowledgements}

The authors wish to thank Dr Ali Yildiz, University of Harran, for his generous support and advice on the study methodology. We also thank Dr Mehmet Gencer and Dr Recep Demirbag, University of Harran, for helping with the literature search strategy. Finally, we are grateful to the referees assigned to this paper for their constructive comments and suggestions that helped improve the manuscript.

Conflict of interest: none declared 


\section{References}

1. Wierzbicki AS. Homocysteine and cardiovascular disease: A review of the evidence. Diab Vasc Dis Res, 2007; 4: 143-502.

2. Yajnik CS, Deshmukh US. Maternal nutrition, intrauterine programming and consequential risks in the offspring. Rev Endocr Metab Disord, 2008; 9: 203-211.

3. Oh R, Brown DL. Vitamin B12 deficiency. Am Fam Physician, 2003; 67: 979-986.

4. Green R. Is it time for vitamin B-12 fortification? What are the questions? Am J Clin Nutr, 2009; 89: 712-716.

5. Ford ES, Smith SJ, Stroup DF, Steinberg KK, Mueller PW, Thacker SB. Homocyst(e)ine and cardiovascular disease: A systematic review of the evidence with special emphasis on case-control studies and nested case-control studies. Int J Epidemiol, 2002; 31: 59-70.

6. Varat MA, Adolph RJ, Fowler NO. Cardiovascular effects of anemia. Am Heart J, 1972; 83: 415-426.

7. Sachdev V, Machado RF, Shizukuda Y et al. Diastolic dysfunction is an independent risk factor for death in patients with sickle cell disease. J Am Coll Cardiol, 2007; 49: 472-479.

8. D'hooge J, Heimdal A, Jamal F et al. Regional strain and strain rate measurements by cardiac ultrasound: Principles, implementation and limitations. Eur J Echocardiogr, 2000; 1: 154-170.

9. Garceau P, Nguyen ET, Carasso S et al. Quantification of myocardial iron deposition by two-dimensional speckle tracking in patients with beta-thalassaemia major and Blackfan-Diamond anaemia. Heart, 2011; 97: 388-393.

10. Artis NJ, Oxborough DL, Williams G, Pepper CB, Tan LB. Two-dimensional SI: A new echocardiographic advance with research and clinical applications. Int J Cardiol, 2008; 123: 240-248.

11. Weidemann F, Jamal F, Kowalski M et al. Can strain rate and strain quantify changes in regional systolic function during dobutamine infusion, B-blockade, and atrial pacing-implications for quantitative stress echocardiography. J Am Soc Echocardiogr, 2002; 15: 416-424.

12. Schiller NB, Shah PM, Crawford M et al. Recommendations for quantitation of the left ventricle by two-dimensional echocardiography. American Society of Echocardiography Committee on Standards, Subcommittee on Quantitation of Two-Dimensional Echocardiograms. J Am Soc Echocardiogr, 1989; 2: 358-367.

13. Nikitin NP, Witte KK. Application of tissue Doppler imaging in cardiology. Cardiology, 2004; 101: 170-184.

14. Akdogan A, Kaya EB, Sahin A et al. Relationship between left ventricular diastolic dysfunction and six minute walk test in patients with systemic sclerosis. Int J Rheum Dis, 2011; 14: 379-383.

15. Di Salvo G, Di Bello V, Salustri A et al. The prognostic value of early left ventricular longitudinal systolic dysfunction in asymptomatic subjects with cardiovascular risk factors. Clin Cardiol, 2011; 34: 500-506.
16. Niedeggen A, Breithardt OA, Franke A. Detection of early systolic dysfunction with strain rate imaging in a patient with light chain cardiomyopathy. Z Kardiol, 2005; 94: 133-136.

17. Lee SW, Choi EY, Jung SY, Choi ST, Lee SK, Park YB. E/E' ratio is more sensitive than $\mathrm{E} / \mathrm{A}$ ratio for detection of left ventricular diastolic dysfunction in patients with systemic sclerosis. Clin Exp Rheumatol, 2010; 28: S12-S17.

18. Yu CM, Sanderson JE, Marwick TH, Oh JK. Tissue Doppler imaging a new prognosticator for cardiovascular diseases. J Am Coll Cardiol, 2007; 49: 1903-1914.

19. Friedberg MK, Mertens L. Tissue velocities, strain, and strain rate for echocardiographic assessment of ventricular function in congenital heart disease. Eur J Echocardiogr, 2009; 10: 585-593.

20. Kimura K, Takenaka K, Ebihara A et al. Speckle Tracking Global Strain Rate E/E' predicts LV filling pressure more accurately than traditional tissue Doppler E/E'. Echocardiography, 2012; 29: 404-410.

21. Mor-Avi V, Lang RM, Badano LP et al. Current and evolving echocardiographic techniques for the quantitative evaluation of cardiac mechanics: ASE/EAE consensus statement on methodology and indications endorsed by the Japanese Society of Echocardiography. J Am Soc Echocardiogr, 2011; 24: 277-313.

22. Pislaru C, Abraham TP, Belohlavek M. Strain and strain rate echocardiography. Curr Opin Cardiol, 2002; 17: 443-454.

23. Taban-Shomal O, Kilter H, Wagner A et al. The cardiac effects of prolonged vitamin B12 and folate deficiency in rats. Cardiovasc Toxicol, 2009; 9: 95-102.

24. Hung J, Beilby JP, Knuiman MW, Divitini M. Folate and vitamin B-12 and risk of fatal cardiovascular disease: Cohort study from Busselton, Western Australia. BMJ, 2003; 326: 131.

25. Looker HC, Fagot-Campagna A, Gunter EW et al. Homocysteine and vitamin $\mathrm{B}(12)$ concentrations and mortality rates in type 2 diabetes. Diabetes Metab Rev, 2007; 23: 193-201.

26. Zeitlin A, Frishman WH, Chee JC. The association of vitamin B12 and folate blood levels with mortality and cardiovascular morbidity incidence in the old old: The Bronx Aging Study. Am J Ther, 1997; 4: 275-281.

27. De Bree A, Verschuren WM, Blom HJ, Nadeau M, Trijbels FJ, Kromhout D. Coronary heart disease mortality, plasma homocysteine, and B-vitamins: a prospective study. Atherosclerosis, 2003; 166: 369-377.

28. Folsom AR, Nieto FJ, McGovern PG et al. Prospective study of coronary heart disease incidence in relation to fasting total homocysteine, related genetic polymorphisms, and B vitamins: The atherosclerosis Risk in communities (ARIC) study. Circulation, 1998; 98: 204-210.

29. Rafnsson SB, Saravanan P, Bhopal RS, Yajnik CS. Is a low blood level of vitamin B12 a cardiovascular and diabetes risk factor? A systematic review of cohort studies. Eur J Nutr, 2011; 50: 97-106.

30. Gueant Rodriguez RM, Spada R, Pooya S et al. Homocysteine predicts increased NT-pro-BNP through impaired fatty acid oxidation. Int J Cardiol, 2012 [Epub ahead of print]. 